INTERNATIONAL JOURNAL OF GYNECOLOGICAL CANCER

(2) OPEN ACCESS

\title{
The upper limit of optimal tumor size in patients with FIGO 2018 stage IB2 cervical cancer undergoing radical hysterectomy
}

\author{
Woo Yeon Hwang (D) , Ju-Hyun Kim, Dong Hoon Suh, Kidong Kim (D), Jae Hong No, \\ Yong Beom Kim
}

Department of Obstetrics and Gynecology, Seoul National University Bundang Hospital, Seongnam, Republic of Korea

\section{Correspondence to} Dr Jae Hong No, Department of Obstetrics and gynecology, Seoul National University Bundang Hospital, Seongnam 13620, Republic of Korea; jhno@snu.ac.kr

Received 9 March 2020 Revised 10 May 2020 Accepted 12 May 2020 Published Online First 28 May 2020

Check for updates

(C) IGCS and ESG0 2020. Re-use permitted under CC BY-NC. No commercial re-use. Published by BMJ.

To cite: Hwang WY, Kim J-H, Suh DH, et al. Int J Gynecol Cancer 2020;30:975-980.

\section{HIGHLIGHTS}

- According to the receiver operating characteristic curve results the optimal cut-off value of tumor size for predicting adjuvant treatment was $2.7 \mathrm{~cm}$.

- In patients with tumor size $\geq 2.7 \mathrm{~cm}$ stage IB2 cervical cancer primary concurrent chemoradiation therapy may be advantageous.

- No significant differences were observed in the progression-free survival and overall survival rates between tumor size smaller or larger than $2.7 \mathrm{~cm}$.

\section{ABSTRACT \\ Objective Patients who undergo radical hysterectomy may require postoperative adjuvant radiotherapy, and all efforts should be made to reduce dual therapy in such patients. The aim of this study was to determine the optimal upper limit of tumor size in patients with International Federation of Gynecology and Obstetrics (FIGO) stage IB2 cervical cancer who undergo radical hysterectomy.}

Methods We retrospectively reviewed the records of 114 patients with FIGO 2018 stage IB2 cervical cancer who underwent primary surgery either with $(n=55)$ or without $(n=59)$ adjuvant radiotherapy from June 2004 to December 2018. The inclusion criteria were as follows: women diagnosed with stage IB2 cervical cancer; primary radical surgery with pelvic lymph node dissection with or without para-aortic lymph node dissection; and patients treated with or without postoperative adjuvant radiation therapy, concurrent chemoradiation therapy, or chemotherapy. A receiver operating characteristic (ROC) curve analysis was used to determine the optimal tumor size cut-off value. The optimal tumor size cut-off value was determined by the maximum sum of sensitivity and specificity.

Results There were 55 and 59 patients treated with or without adjuvant therapy, respectively, after radical hysterectomy. Age, histologic type, and pelvic and paraaortic lymph node sampling/dissection status were similar between each group. The number of patients with a tumor size $<2.7 \mathrm{~cm}$ and $\geq 2.7 \mathrm{~cm}$ was 39 and 75 , respectively. The decision for adjuvant treatment after radical hysterectomy in patients with stage IB2 cervical cancer was influenced by intermediate risk factors (lymphovascular space invasion, $23.7 \%$ vs $76.4 \%, p<0.001$; deep $1 / 3$ of invasion, $16.9 \%$ vs $61.8 \%, p<0.001$ ) and high risk factors (lymph node metastasis, $0 \%$ vs $40.0 \%, p<0.001$; involvement of parametrium, $1.7 \%$ vs $16.4 \%, p=0.007$ ). According to the ROC curve results considering the best sensitivity and specificity, the optimal cut-off value of tumor size for predicting adjuvant treatment was $2.7 \mathrm{~cm}$ (sensitivity 0.85 , specificity 0.52 ). The number of patients with a tumor size
$<2.7 \mathrm{~cm}$ and $\geq 2.7 \mathrm{~cm}$ was $39(34.2 \%)$ and $75(65.8 \%)$, respectively. No significant differences were observed in the progression-free survival $(p=0.22)$ and overall survival $(p=0.28)$ rates between tumor size smaller than $2.7 \mathrm{~cm}$ and larger than $2.7 \mathrm{~cm}$.

Conclusions A cervical tumor larger than $2.7 \mathrm{~cm}$ before radical surgery in stage IB2 may predispose to potential complications from combining radical hysterectomy and concurrent chemoradiation,. We consider that concurrent chemoradiation therapy is a more appropriate choice for tumor size over $2.7 \mathrm{~cm}$ per the revised FIG0 2018 criteria for stage IB2 cervical cancer.

\section{INTRODUCTION}

In 2018, the International Federation of Gynecology and Obstetrics (FIGO) revised the staging system for cervical cancer. One of the major changes from the 2014 FIGO staging criteria was that stage IB cervical cancer is now divided into three subgroups including an additional cut-off at $2 \mathrm{~cm}$ : stage IB1 $(<2 \mathrm{~cm})$, stage IB2 $(\geq 2 \mathrm{~cm}$ to $<4 \mathrm{~cm})$, and stage IB3 $(\geq 4 \mathrm{~cm}) .^{1-3}$ Another major change in the updated FIGO staging criteria is the incorporation of imaging and pathological findings. ${ }^{1-3}$ Although the staging and treatment recommendations in each stage have been revised according to the 2018 FIGO staging criteria, much of the data that have been reported utilized the previous 2009 FIGO staging system. ${ }^{4}$ Subsequently, new research studies should be carried out with the revised staging system. At present, there are two available treatment strategies for the treatment of FIG0 2018 stage IB2 cervical cancer criteria: primary surgery consisting of radical hysterectomy plus bilateral pelvic lymph node dissection with or without para-aortic lymph node dissection or combined pelvic external beam radiotherapy, and brachytherapy with or without concurrent chemoradiation therapy. ${ }^{4}$ For 


\section{Original research}

stage IB2 cervical cancer, identical treatment outcomes were found in primary radiation versus surgery with or without postoperative radiation therapy. 256

Adjuvant therapy is recommended in surgically treated patients who have high-risk pathologic factors, including positive lymph node, positive surgical margins, and involvement of parametrium. ${ }^{7}$ In addition, a large tumor size, deep stromal invasion, and lymphovascular space invasion are regarded as intermediate risk factors for cervical cancer recurrence. Patients with these risk factors are also candidates for postoperative adjuvant therapy to improve the recurrence rate based on the results of randomized studies reported by Sedlis et al. ${ }^{7-9}$ However, there have been concerns regarding the potential complications and toxicity related to the combination of surgery and chemoradiotherapy, such as gastrointestinal toxicity and urological complications. ${ }^{510} 11$ Therefore, some clinicians recommend concurrent chemoradiation therapy as a primary treatment and avoid surgery for those who may require adjuvant postoperative radiation. ${ }^{12}$ If the need for postoperative concurrent chemoradiation therapy could be predicted, then concurrent chemoradiation therapy rather than surgery could be selected as the primary treatment option. Subsequently, the morbidity rate may be reduced by avoiding combined therapies.

Tumor size is one of the important risk factors that could be measured by imaging exams before initiating treatment. In this retrospective study, we investigated whether tumor size could be a predicting factor for those who may need adjuvant treatment after surgery in patients with stage IB2 cervical cancer. As a result, the most ideal primary treatment can be selected for certain groups of patients with stage IB2 cervical cancer per the revised 2018 FIG0 staging criteria.

\section{METHODS}

This study was approved by the institutional review board of the Seoul National University Bundang Hospital (No. B-2001-588-103) and performed in accordance with the principles of the Declaration of Helsinki. The requirement for informed consent was waived. We retrospectively reviewed the medical records of 114 patients with revised 2018 FIG0 stage IB2 cervical cancer who were treated at Seoul National University Bundang Hospital, a tertiary hospital in Korea, from June 2004 to December 2018. All patients were regrouped based on the new FIGO 2018 staging system. The inclusion criteria were as follows: women who were diagnosed with FIG0 2018 stage IB2 cervical cancer according to radiologic findings; primary radical surgery with pelvic lymph node dissection with or without para-aortic lymph node dissection; and patients treated with or without postoperative adjuvant radiation therapy, concurrent chemoradiation therapy, or chemotherapy. The exclusion criteria were as follows: patients who were initially treated with therapy other than surgery such as concurrent chemoradiation therapy, radiation therapy, or chemotherapy; patients who had an incomplete treatment; patients who had insufficient clinical and pathologic data; patients who were diagnosed incidentally during the operation with other disease in addition to cervical cancer; and patients treated with neoadjuvant chemotherapy.

We reviewed the medical records of patients; imaging data from magnetic resonance imaging (MRI), computed tomography (CT), and positron emission tomography/CT; operation records; pathological findings; clinicopathologic characteristics; and adjuvant treatment status. The initial tumor size of 111 patients were measured by $\mathrm{MRI}$ and three patients by CT before undergoing primary surgery. In addition, as we recategorized the patients with the new staging system, we used image findings to select patients with the tumor size $\geq 2 \mathrm{~cm}$ to $<4 \mathrm{~cm}$ and to exclude patients with retroperitoneal lymph nodes metastasis, the factor which was not included in the previous staging system. The risk factors such as lymphovascular space invasion, depth of invasion, lymph node metastasis, involvement of parametrium, and involvement of resection margin were evaluated by pathological findings. During the study period, five surgeons performed all of the operations in Seoul National University Bundang Hospital faculties. Type III hysterectomy was performed either by a laparoscopic or open approach according to the surgeon's decision.

We evaluated for differences in clinicopathologic characteristics between two groups: patients who underwent radical surgery, and those who underwent surgery following concurrent chemoradiation therapy. The Student's t-test and Mann-Whitney $U$ test were performed for comparisons of continuous variables. Pearson's $\chi^{2}$ test or Fisher's exact test were performed to compare categorical variables. A receiver operating characteristic $(\mathrm{ROC})$ curve analysis was used to investigate which size parameter is best suited for the prediction of adjuvant treatment after radical hysterectomy for patients with stage IB2 cervical cancer per the revised 2018 FIGO staging criteria. The optimal tumor size cut-off value was determined by the maximum sum of sensitivity and specificity. Pearson's correlation coefficient was calculated to elucidate the correlation between radiologic tumor size before surgery and pathologic tumor size after surgery. All analyses were performed using Statistical Package for the Social Sciences (SPSS) software for Windows (version 25.0; SPSS Inc, Chicago, IL). A value of $p<0.05$ indicated statistical significance.

\section{RESULTS}

Of the 114 patients, 55 were treated with and 59 were treated without adjuvant treatment, respectively, after radical hysterectomy. The overall patient characteristics are shown in Table 1. Age, histologic type, and pelvic and para-aortic lymph node sampling/ dissection status were similar between each group. The preoperative tumor size by MRI was $3.1 \pm 0.4 \mathrm{~cm}$ and $2.6 \pm 0.5 \mathrm{~cm}$ in patients treated with and without adjuvant treatment, respectively. The number of patients who underwent open abdominal radical hysterectomy were significantly higher in the adjuvant treatment group (44.1\% vs $67.3 \%, p=0.013)$. The decision for adjuvant treatment after radical hysterectomy in patients with stage IB2 cervical cancer was influenced by intermediate risk factors (lymphovascular space invasion, $23.7 \%$ vs $76.4 \%$, $p<0.001$; deep $1 / 3$ of invasion, $16.9 \%$ vs $61.8 \%, p<0.001$ ) and high risk factors (lymph node metastasis, $0 \%$ vs $40.0 \%, p<0.001$; involvement of parametrium, $1.7 \%$ vs $16.4 \%, p=0.007$ ), except for those who had involvement of resection margin ( $0 \%$ vs $1.8 \%, p=0.48)$ according to pathology reports.

Tumor size was selected to calculate the probability of adjuvant treatment after primary hysterectomy. We used a ROC curve to assess the optimal cut-off value of tumor size in patients with 
Table 1 Clinical and pathologic characteristics of study population

\begin{tabular}{|c|c|c|c|c|}
\hline Characteristics & $\begin{array}{l}\text { Overall } \\
(n=114)\end{array}$ & $\begin{array}{l}\text { Adjuvant treatment }(-) \\
(\mathrm{n}=59)\end{array}$ & $\begin{array}{l}\text { Adjuvant treatment }(+) \\
(n=55)\end{array}$ & $P$ value \\
\hline Age, years & $46.8 \pm 10.5$ & $45.8 \pm 11.0$ & $47.9 \pm 10.0$ & $0.283^{*}$ \\
\hline Histologic type & & & & $0.139^{*}$ \\
\hline Squamous cell carcinoma & $68(59.6)$ & $31(52.5)$ & 37 (67.3) & \\
\hline Adenocarcinoma & $35(30.7)$ & $23(39.0)$ & $12(21.8)$ & \\
\hline Others & $11(9.6)$ & $5(8.5)$ & $6(10.9)$ & \\
\hline Initial tumor size, $\mathrm{cm}$ & $2.9 \pm 0.5$ & $2.6 \pm 0.5$ & $3.1 \pm 0.4$ & $<0.001^{*}$ \\
\hline Operation method & & & & $0.013^{*}$ \\
\hline Minimally invasive surgery & $51(44.7)$ & 33 (55.9) & $18(32.7)$ & \\
\hline Open & $63(55.3)$ & $26(44.1)$ & $37(67.3)$ & \\
\hline Pelvic lymph node & & & & $>0.999 \dagger$ \\
\hline No & $1(0.9)$ & $1(1.7)$ & $0(0)$ & \\
\hline Sampling/dissection & $113(99.1)$ & 58 (98.3) & $55(100.0)$ & \\
\hline Para-aortic lymph node & & & & $0.074^{*}$ \\
\hline No & $89(78.1)$ & $50(84.7)$ & 39 (70.9) & \\
\hline Sampling/dissection & $25(21.9)$ & $9(15.3)$ & $16(29.1)$ & \\
\hline \multicolumn{5}{|l|}{ Risk factors } \\
\hline Lymphovascular space invasion & & & & $<0.001^{*}$ \\
\hline Negative & $58(50.9)$ & 45 (76.3) & $13(23.6)$ & \\
\hline Positive & $56(49.1)$ & $14(23.7)$ & $42(76.4)$ & \\
\hline Depth of Invasion & & & & $<0.001^{*}$ \\
\hline Superficial $1 / 3$ & $22(19.3)$ & 20 (33.9) & $2(3.6)$ & \\
\hline Middle $1 / 3$ & $48(42.1)$ & $29(49.2)$ & $19(34.5)$ & \\
\hline Deep 1/3 & $44(38.6)$ & $10(16.9)$ & $34(61.8)$ & \\
\hline Lymph node metastasis & & & & $<0.001^{*}$ \\
\hline Negative & $92(80.7)$ & $59(100.0)$ & $33(60.0)$ & \\
\hline Positive & $22(19.3)$ & $0(0)$ & $22(40.0)$ & \\
\hline Parametrium involvement & & & & $0.007 \dagger$ \\
\hline Negative & $104(91.2)$ & $58(98.3)$ & $46(83.6)$ & \\
\hline Positive & $10(8.8)$ & $1(1.7)$ & $9(16.4)$ & \\
\hline Resection margin involvement & & & & $0.487 \dagger$ \\
\hline Negative & $113(99.1)$ & $59(100.0)$ & $54(98.2)$ & \\
\hline Positive & $1(0.9)$ & $0(0)$ & $1(1.8)$ & \\
\hline
\end{tabular}

Values are presented as mean \pm SD or $\mathrm{n}(\%)$.

${ }^{*} P$ values were calculated by Pearson's $\chi^{2}$ test.

$\dagger P$ values were calculated by Fisher's exact test.

cervical cancer treated with radical hysterectomy (Figure 1). According to the ROC curve results considering the best sensitivity and specificity, the optimal cut-off value of tumor size for predicting adjuvant treatment was $2.7 \mathrm{~cm}$ (sensitivity 0.85 , specificity 0.52). In a ROC curve analysis, the tumor size for predicting adjuvant treatment was statistically significant (area under the curve $0.73,95 \% \mathrm{Cl} 0.63$ to $0.82 ; \mathrm{p}=0.047$ ). The number of patients with a tumor size $<2.7 \mathrm{~cm}$ and $\geq 2.7 \mathrm{~cm}$ was $39(34.2 \%)$ and 75 $(65.8 \%)$, respectively. Intermediate and high-risk factors of the two groups are compared in Table 2. According to the involvement of lymphovascular space invasion, deep $1 / 3$ of stromal invasion, and pelvic lymph node metastasis status in the pathologic findings, the proportion of patients who had positive findings were significantly higher in those with a tumor size $\geq 2.7 \mathrm{~cm}$ (lymphovascular space invasion, $25.6 \%$ vs $59.3 \%, p<0.001$; deep $1 / 3$ of stromal invasion, $17.9 \%$ vs $49.3 \%, p=0.003$; lymph node metastasis, $5.1 \%$ vs $26.7 \%, p=0.006$; involvement of parametrium, $1.7 \%$ vs $16.4 \%, p=0.007)$. The involvements of parametrium and resection margin were not significantly different between the two groups (parametrium involvement, $5.1 \%$ vs $26.7 \%, \mathrm{p}=0.49$; resection margin involvement, $0 \%$ vs $1.3 \%, p>0.99$ ). Adjuvant treatment was administered in six (17.6\%) and $50(61.7 \%)$ patients with an initial 


\section{Original research}

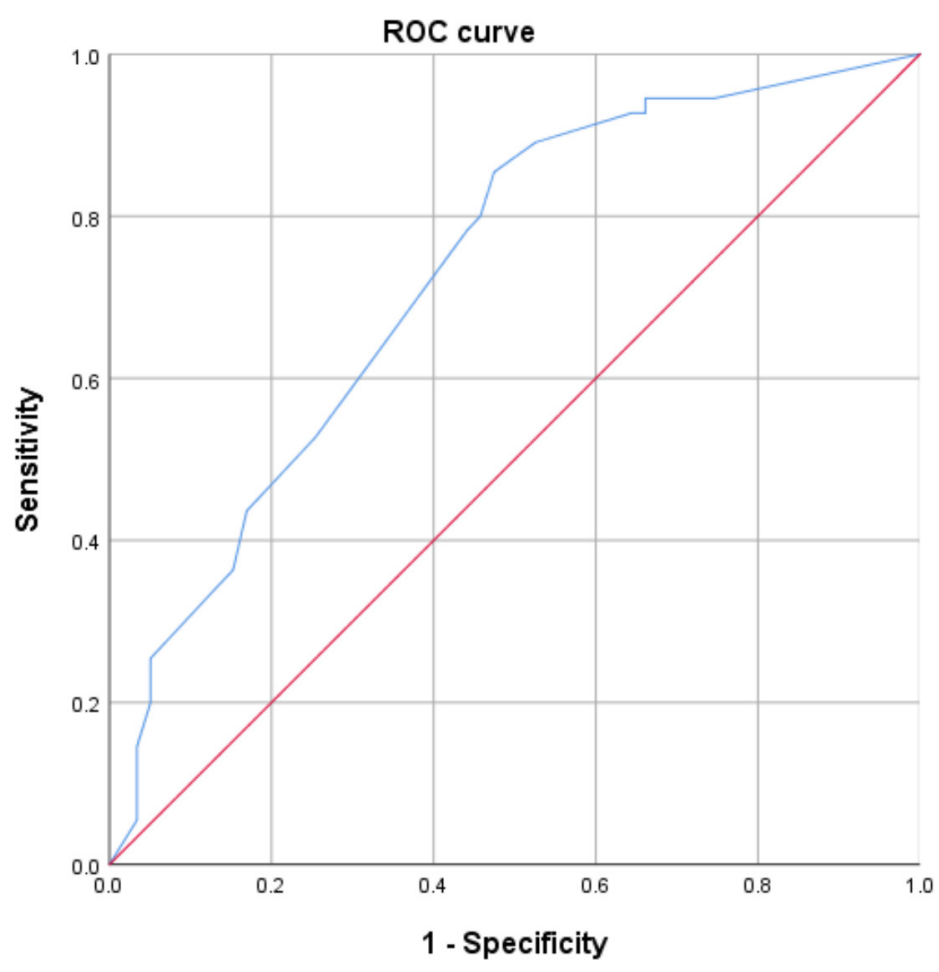

Figure 1 Receiver operating characteristic $(\mathrm{ROC})$ curve analysis of tumor size prediction according to adjuvant treatment status after radical hysterectomy in patients with cervical cancer. The area under the curve was 0.728 ( $95 \% \mathrm{Cl} 0.635$ to 0.820 ; $\mathrm{p}=0.047$ ), and $2.7 \mathrm{~cm}$ was determined as the best tumor size cut-off value for predicting the need for adjuvant treatment.

tumor size $<2.7 \mathrm{~cm}$ and $\geq 2.7 \mathrm{~cm}$, respectively. Our study showed that, compared with patients with no adjuvant therapy after radical hysterectomy, those with adjuvant concurrent chemoradiation therapy among tumor size $\geq 2.7 \mathrm{~cm}$ implied an odds ratio of 6.50 (95\% Cl 2.63 to 16.11).

The tumor size on radiologic findings before surgery and pathological tumor size after surgery showed a positive linear relationship.
Pearson's correlation coefficient was calculated as 0.50 , which is considered moderately correlated with statistical significance $(\mathrm{p}<0.001)$. During a median length of follow-up of 55.6 months (range 1.3-164.7), 14 patients (12.3\%) experienced disease recurrence and three patients $(2.6 \%)$ died. No significant differences were observed in the progression-free survival $(p=0.224)$ and overall survival $(p=0.283)$ rates between tumor size smaller than $2.7 \mathrm{~cm}$ and larger than $2.7 \mathrm{~cm}$.

Table 2 Comparison of intermediate and high-risk factors between those with initial tumor sizes $<2.7 \mathrm{~cm}$ and $\geq 2.7 \mathrm{~cm}$ defined by preoperative magnetic resonance imaging

\begin{tabular}{|c|c|c|c|}
\hline Characteristics & $\begin{array}{l}\text { Initial tumor size }<2.7 \mathrm{~cm} \\
(\mathrm{n}=39)\end{array}$ & $\begin{array}{l}\text { Initial tumor size } \geq 2.7 \mathrm{~cm} \\
(\mathrm{n}=75)\end{array}$ & $P$ value \\
\hline \multicolumn{4}{|l|}{ Risk factors } \\
\hline \multicolumn{4}{|l|}{ Intermediate risk } \\
\hline Lymphovascular space invasion & $10(25.6)$ & $46(59.3)$ & $<0.001^{*}$ \\
\hline Depth of Invasion & & & $0.003^{*}$ \\
\hline Superficial $1 / 3$ & $12(30.8)$ & $10(13.3)$ & \\
\hline Middle $1 / 3$ & $20(51.3)$ & $28(37.3)$ & \\
\hline Deep 1/3 & $7(17.9)$ & $37(49.3)$ & \\
\hline \multicolumn{4}{|l|}{ High risk } \\
\hline Lymph node metastasis & $2(5.1)$ & $20(26.7)$ & $0.006^{*}$ \\
\hline Parametrium involvement & $2(5.1)$ & $8(10.7)$ & $0.490 \dagger$ \\
\hline Resection margin involvement & $0(0.0)$ & $1(1.3)$ & $>0.999 \dagger$ \\
\hline Adjuvant treatment & $6(17.6)$ & $50(61.7)$ & $<0.001^{*}$ \\
\hline
\end{tabular}

Values are presented as $\mathrm{n}(\%)$.

*P values were calculated by Pearson's $\chi^{2}$ test.

†P values were calculated by Fisher's exact test. 


\section{DISCUSSION}

Our results demonstrate that stage IB2 cervical cancer patients with a tumor size larger than $2.7 \mathrm{~cm}$ before radical surgery may be predisposed to potential complications from combining radical hysterectomy and concurrent chemoradiation. We consider that concurrent chemoradiation therapy is a more appropriate choice for tumor size over $2.7 \mathrm{~cm}$ for FIGO 2018 stage IB2 cervical cancer.

There is a lack of evidence for the best treatment option in patients with stage IB2 cervical cancer per the revised 2018 FIG0 staging criteria. The therapeutic strategies for early stage cervical cancer includes radical surgery, radiation therapy, or concurrent chemoradiation therapy depending on the FIGO stage, fertility preservation, the patient's performance status, and the surgeon's clinical decision. ${ }^{41314}$ According to the guidelines by the National Comprehensive Cancer Network, radical hysterectomy with bilateral pelvic lymph node dissection with or without para-aortic lymph node dissection is one of the preferred treatment options for patients with FIGO 2018 stage IB2 cervical cancer. ${ }^{4515}$ Another possible option is combined pelvic external beam radiation therapy and brachytherapy with or without concurrent platinum-containing chemotherapy. ${ }^{5}$

One of the advantages of primary surgery is that one can obtain a histopathologic outcome associated with risk factors and final surgical staging from the surgical specimen. ${ }^{16}$ In addition, it is possible to treat some cervical cancers that develop resistance to radiation therapy. ${ }^{16}$ Furthermore, when pelvic recurrence occurs, it can be successfully cured by radiation therapy in those patients who are primarily treated with radical hysterectomy alone, whereas salvage surgery in a radiated pelvis is challenging and associated with higher surgical complications such as permanent ostomies for colon and urinary bladder. ${ }^{17}$

Following primary radical hysterectomy, adjuvant treatment is indicated depending on pathologic risk factors and disease stage. Radiation therapy or concurrent chemoradiation therapy is recommended for patients who have high risk factors (positive lymph node, positive surgical margins, and involvement of parametrium) or various combinations of intermediate risk factors (large tumor size, deep stromal invasion, and lymphovascular space invasion) that meet the Sedlis criteria. ${ }^{8}{ }^{18-20}$ The combination of surgery and radiotherapy has higher morbidity and complications. ${ }^{5}$ Therefore, if the need for adjuvant treatment could be predicted following radical surgery, one could appropriately select patients for primary surgery by stratifying their risks and needs for additional postoperative chemoradiation treatments. Furthermore, it would be an acceptable option for reducing surgical morbidity by avoiding combined therapy.

Among the risk factors that require additional therapy after surgery, tumor size is the only risk factor that could be precisely measured by clinical imaging studies before surgery without a pathologic report, while the other factors are determined pathologically after radical hysterectomy. Thus, we selected tumor size for predicting adjuvant treatment status. Among the diagnostic imaging tools, MRI has $90 \%$ sensitivity, $98 \%$ specificity, and up to $95 \%$ accuracy for stage IB or higher with primary tumors over $1 \mathrm{~cm}$ in size. ${ }^{21-23}$ Other imaging studies including ultrasound, CT, and positron emission tomography based on local resources provide comparable information but have limitations, especially in the detection of the primary tumor and parametrial involvement. ${ }^{24} 25$
There are several strengths of this study. First, we regrouped all patients based on the new 2018 FIGO system. Second, it enhances counseling when suggesting treatment options of primary surgery or chemoradiation based on their tumor size for stage IB2 cervical cancer patients, as there is no high-certainty evidence between treatment options. Finally, we measured the tumor size in most patients using MRI which has high accuracy. Ideally, with the availability of non-invasive diagnostic imaging tools, it may be possible to recommend appropriate treatment options avoiding combination therapies. Limitations of the current study include its retrospective nature, the lack of data on toxicities associated with adjuvant radiotherapy, and the small number of patients. Further randomized trials are required to explore the survival impact and local control rate of different treatment modalities based on the revised stage $1 \mathrm{~B} 2$ cervical cancer.

Stage IB2 cervical cancer can be treated successfully in most patients with either primary radical surgery with or without adjuvant treatment or primary concurrent chemoradiation therapy. In order to minimize the complication of dual treatment, a judicious selection is necessary before planning primary radical surgery. This may be especially important for patients who have difficulties enduring surgery. We re-evaluated the current treatment methods by tumor size for patients with stage IB2 cervical cancer. Although a primary surgical approach has some advantages over primary concurrent chemoradiation therapy, we consider that physicians should propose a primary concurrent chemoradiation therapy for patients with a cervical tumor $\geq 2.7 \mathrm{~cm}$. Furthermore, physicians should also counsel the patients with stage IB2 cervical cancer regarding the uncertainty of treatment options and potential adverse events related to each treatment, especially for those with tumors larger than $2.7 \mathrm{~cm}$ who have increased risk of adjuvant therapy after primary surgery.

\section{Twitter Kidong Kim @KIDONG_KIM_MD}

Contributors Conceptualization: WYH, JHN. Methology: WYH, JHN. Data acquisition: WYH. Validation: WYH, JHN. Formal analysis and investigation: all authors. Writing - original draft: WYH. Writing - review \& editing: all authors. Supervision: JHN.

Funding The authors have not declared a specific grant for this research from any funding agency in the public, commercial or not-for-profit sectors.

Competing interests None declared.

Patient consent for publication Not required.

Provenance and peer review Not commissioned; externally peer reviewed.

Data availability statement All data relevant to the study are included in the article or uploaded as supplementary information.

Open access This is an open access article distributed in accordance with the Creative Commons Attribution Non Commercial (CC BY-NC 4.0) license, which permits others to distribute, remix, adapt, build upon this work non-commercially, and license their derivative works on different terms, provided the original work is properly cited, an indication of whether changes were made, and the use is noncommercial. See: http://creativecommons.org/licenses/by-nc/4.0/.

\section{ORCID iDs}

Woo Yeon Hwang http://orcid.org/0000-0003-0231-8330

Kidong Kim http://orcid.org/0000-0001-9254-6024

\section{REFERENCES}

1 Berek JS, Matsuo K, Grubbs BH, et al. Multidisciplinary perspectives on newly revised $2018 \mathrm{FIGO}$ staging of cancer of the cervix uteri. $J$ Gynecol Oncol 2019;30:e40. 


\section{Original research}

2 Bhatla N, Aoki D, Sharma DN, et al. Cancer of the cervix uteri. Int J Gynaecol Obstet 2018;143(Suppl 2):22-36.

3 Bhatla N, Berek JS, Cuello Fredes M, et al. Revised FIGO staging for carcinoma of the cervix uteri. Int J Gynecol Obstet 2019;145:129-35.

4 National Comprehensive Cancer Network. Clinical practice guideline in oncology, cervical cancer (version 5), 2019.

5 Landoni F, Maneo A, Colombo A, et al. Randomised study of radical surgery versus radiotherapy for stage Ib-Ila cervical cancer. Lancet 1997;350:535-40.

6 Morley GW, Seski JC. Radical pelvic surgery versus radiation therapy for stage I carcinoma of the cervix (exclusive of microinvasion). Am J Obstet Gynecol 1976;126:785-98.

7 Jewell EL, Kulasingam S, Myers ER, et al. Primary surgery versus chemoradiation in the treatment of IB2 cervical carcinoma: a cost effectiveness analysis. Gynecol Oncol 2007;107:532-40.

8 Sedlis A, Bundy BN, Rotman MZ, et al. A randomized trial of pelvic radiation therapy versus no further therapy in selected patients with stage IB carcinoma of the cervix after radical hysterectomy and pelvic lymphadenectomy: a Gynecologic Oncology Group study. Gynecol Oncol 1999;73:177-83.

9 Rotman M, Sedlis A, Piedmonte MR, et al. A phase III randomized trial of postoperative pelvic irradiation in stage IB cervical carcinoma with poor prognostic features: follow-up of a Gynecologic Oncology Group study. Int J Radiat Oncol Biol Phys 2006;65:169-76.

10 Nama V, Angelopoulos G, Twigg J, et al. Type II or type III radical hysterectomy compared to chemoradiotherapy as a primary intervention for stage IB2 cervical cancer. Cochrane Database Syst Rev 2018;10.

11 Roszak A, Wareńczak-Florczak Z, Bratos K, et al. Incidence of radiation toxicity in cervical cancer and endometrial cancer patients treated with radiotherapy alone versus adjuvant radiotherapy. Rep Pract Oncol Radiother 2012;17:332-8.

12 Lapuz C, Kondalsamy-Chennakesavan S, Bernshaw D, et al. Stage IB cervix cancer with nodal involvement treated with primary surgery or primary radiotherapy: patterns of failure and outcomes in a contemporary population. J Med Imaging Radiat Oncol 2016;60:274-82.

13 Hsieh H-Y, Huang J-W, Lu C-H, et al. Definite chemoradiotherapy is a competent treatment option in FIGO stage IB2 cervical cancer compared with radical surgery +/- neoadjuvant chemotherapy. J Formos Med Assoc 2019;118:99-108.
14 Seki T, Tanabe H, Nagata C, et al. Adjuvant therapy after radical surgery for stage IB-IIB cervical adenocarcinoma with risk factors. Jpn J Clin Oncol 2017;47:32-8.

15 Landoni F, Maneo A, Cormio G, et al. Class II versus class III radical hysterectomy in stage IB-IIA cervical cancer: a prospective randomized study. Gynecol Oncol 2001;80:3-12.

16 Moreno-Acosta P, Vallard A, Carrillo S, et al. Biomarkers of resistance to radiation therapy: a prospective study in cervical carcinoma. Radiat Oncol 2017;12.

17 Gadducci A, Tana R, Cosio S, et al. Treatment options in recurrent cervical cancer (review). Oncol Lett 2010;1:3-11.

18 Monk BJ, Wang J, Im S, et al. Rethinking the use of radiation and chemotherapy after radical hysterectomy: a clinical-pathologic analysis of a Gynecologic Oncology Group/Southwest Oncology Group/Radiation Therapy Oncology Group trial. Gynecol Oncol 2005;96:721-8.

19 Yan M, Zhang Y-N, He J-H, et al. Influence of lymph vascular space invasion on prognosis of patients with early-stage cervical squamous cell carcinoma. Chin J Cancer 2010;29:425-30.

20 Marchiolé $P$, Buénerd A, Benchaib $M$, et al. Clinical significance of lymphovascular space involvement and lymph node micrometastases in early-stage cervical cancer: a retrospective case-control surgico-pathological study. Gynecol Oncol 2005;97:727-32.

21 Mitchell DG, Snyder B, Coakley F, et al. Early invasive cervical cancer: tumor delineation by magnetic resonance imaging, computed tomography, and clinical examination, verified by pathologic results, in the ACRIN 6651/GOG 183 intergroup study. $J$ Clin Oncol 2006;24:5687-94.

22 Zand $\mathrm{KR}$, Reinhold $\mathrm{C}$, Abe $\mathrm{H}$, et al. Magnetic resonance imaging of the cervix. Cancer Imaging 2007;7:69-76.

23 Otero-García MM, Mesa-Álvarez A, Nikolic O, et al. Role of MRI in staging and follow-up of endometrial and cervical cancer: pitfalls and mimickers. Insights Imaging 2019;10:19.

24 Bipat S, Glas AS, van der Velden J, et al. Computed tomography and magnetic resonance imaging in staging of uterine cervical carcinoma: a systematic review. Gynecol Oncol 2003;91:59-66.

25 Epstein E, Testa A, Gaurilcikas A, et al. Early-stage cervical cancer: tumor delineation by magnetic resonance imaging and ultrasound - a European multicenter trial. Gynecol Oncol 2013;128:449-53. 